\title{
Curriculum Ideological and Political of Higher Mathematics-Taking Extreme Value as an Example
}

\author{
Zhonghui Xue*, Yuemin Teng, Qianfeng Ma \\ Shanghai Publishing and Printing College, Shanghai, China \\ Email: *hnlgxzh@163.com
}

How to cite this paper: Xue, Z.H., Teng, Y.M. and Ma, Q.F. (2020) Curriculum Ideological and Political of Higher Mathematics-Taking Extreme Value as an Example. Open Access Library Journal, 7: e6858.

https://doi.org/10.4236/oalib.1106858

Received: September 24, 2020

Accepted: October 26, 2020

Published: October 29, 2020

Copyright () 2020 by author(s) and Open Access Library Inc.

This work is licensed under the Creative Commons Attribution International License (CC BY 4.0).

http://creativecommons.org/licenses/by/4.0/

\begin{abstract}
Based on the teaching content of extreme value of higher mathematics, this paper integrates knowledge teaching and moral education through "Three-implicit and Three-fusional". Through the interpretation of the ideological value of extreme value knowledge points, it integrates knowledge teaching and value guidance organically, guides students to behave correctly, do things, and help students develop in an all-round way.
\end{abstract}

\section{Subject Areas}

Education

\section{Keywords}

Higher Mathematics, Extreme, Curriculum Ideological and Political, Three-Implicit and Three-Fusional

\section{1. 前言}

习近平总书记在 2016 年的全国高校思想政治工作中明确提出, “其他各 门课都要守好一段渠、种好责任田, 使各类课程与思想政治理论课同向同行, 形成协同效应” ，从而要 “把思想政治工作贯穿教育教学全过程, 实现全程 育人、全方位育人，努力开创我国高等教育事业发展新局面”。“课程思政” 这一概念早在 2014 年就由上海市教育委员会提出, 并在教育部的指导下在上 海高校进行了试验, 取得了创新性的突破, 习近平总书记的讲话精神为课程 思政的向纵深发展提供了强有力的思想支撑和方向指引。布庸置疑, 课程思 政是新时代思想政治教育发展的重要方向，理解和把握新时代课程思政的内 涵, 并积极寻求探索实践是新时代赋予教师的光荣使命，对于培养 “四个服 
务” 人才, 进而实现中华民族伟大复兴的中国梦具有十分重要的意义。在融 入方式、融入范式的探索方面, 上海出版印刷高等专科学校课程思政的 “三 寓三式” 具有较为重要的借鉴意义[1] [2]。

高等数学是高校所有理工科、经济管理科等专业的公共必修基础课, 贯 穿整个大学第一学年, 正是学生从高中进入大学的过渡, 是熟悉大学学习的 关键, 同时高等数学蕴含的辩证思想和思维, 以及正确的处理问题的和解决 问题对的方式等对于奠定学生思想教育基调甚为关键, 可以说是树立科学的 世界观和价值观的重要时间点。正如习近平总书记所言, 青少年学生正处于 人生的 “拔节孕穗期”, 最需要精心引导和栽培, 而且青少年的价值取向在 某种程度上决定了未来整个社会的价值取向, 因此抓好这一时期的价值观教 育十分重要。如何在高等数学的教学过程中贯彻课程思政, 是高等数学课程 教学中面临的一个新的问题, 也是高等数学任课教师践行习总书记 “同向同 行” 精神的必然选择。众所周知, 高等数学以高度的抽象思维和逻辑思维著 称, 而抽象思维和罗辑思维的基础是正确的世界观和方法论, 因此在高等数 学中蕴含着丰富的德育元素, 如在高等数学导数应用章节, 有关极值的知识 点蕴含着大量的价值观、利益观、义利观和发展观的德育元素。

本文分 5 个部分: 第 $1 、 2$ 部分主要对极值和最值在数学中的概念作简单 介绍; 第 3 部分主要探究了极值和最值的数学思想内涵; 第 4 部分探究了极 值和最值的思想教育价值; 第 5 部分给出了总结性的论述, 期望能够对从事 课程思政研究的同仁提供一些参考。

\section{2. 极值知识概述}

\section{1. 函数的极小值点和极小值的概念}

函数 $y=f(x)$ 在点 $x=a$ 的函数值 $f(a)$ 比它在点 $x=a$ 附近其他点的函 数值都小, $f^{\prime}(a)=0$; 而且在点 $x=a$ 附近的左侧 $f^{\prime}(x)<0$, 右侧 $f^{\prime}(x)>0$, 则点 $a$ 叫做函数 $y=f(x)$ 的极小值点, $f(a)$ 叫做函数 $y=f(x)$ 的极小值。如 图 1(a)所示。

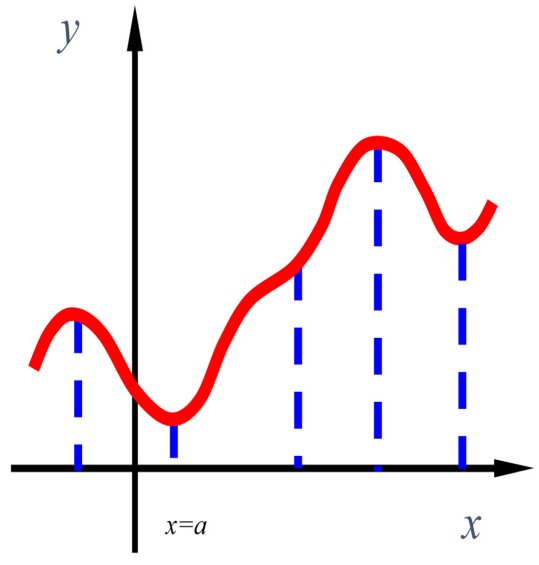

(a)

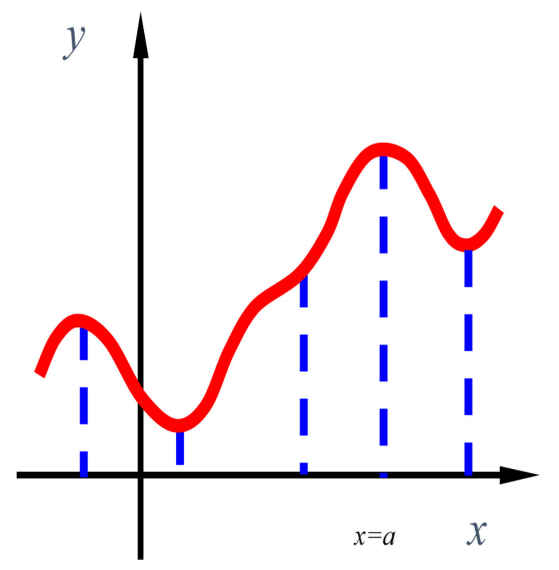

(b)

图 1. (a) 对应于 $x=a$ 时的极小值示意图; (b) 对应于 $x=a$ 时的极大值示意图 


\section{2. 函数的极大值点和极大值的概念}

函数 $y=f(x)$ 在点 $x=b$ 的函数值 $f(b)$ 比它在点 $x=b$ 附近其他点的函 数值都大, $f^{\prime}(b)=0$; 而且在点 $x=b$ 附近的左侧 $f^{\prime}(x)>0$, 右侧 $f^{\prime}(x)<0$, 则点 $b$ 叫做函数 $y=f(x)$ 的极大值点, $f(b)$ 叫做函数 $y=f(x)$ 的极大值。 极小值点、极大值点统称为极值点, 极大值和极小值统称为极值。如图 1(b) 所示。

\section{3. 求函数极值的步骤}

(1) 求导数 $f^{\prime}(x)$;

(2) 求驻点和不可导点, 即 $f^{\prime}(x)=0$ 的根和函数 $y=f(x)$ 的不可导点;

(3) 检查导数在驻点和不可导点左右的导数值的符号, 如果左正右负, 那 么 $y=f(x)$ 在这个点(驻点或不可导点)处取极大值, 如果左负右正, 那么 $y=f(x)$ 在这个驻点或不可导点处取极小值。

\section{4. 求函数的最大值与最小值的步骤}

在闭区间 $[a, b]$ 上连续, 在 $(a, b)$ 内可导, $f(x)$ 在 $[a, b]$ 上一定有最大值 和最小值, 求最大值与最小值的步骤:

(1) 求 $f(x)$ 在 $(a, b)$ 内的极值;

(2) 将 $f(x)$ 的各极值与 $f(a), f(b)$ 比较, 其中最大的一个是最大值, 最小的一个是最小值。

\section{3. 极值和最值的知识点比较分析}

\section{1. 关于极值和最值}

极值和最值的知识点比较如表 1 所示, 需特别说明的是:

1) 函数的最大值和最小值是一个整体性概念, 最大值必须是整个区间上 所有函数值中的最大值, 最小值必须是整个区间上所有函数值中的最小值。

2) 函数的最大值、最小值是通过比较整个定义区间的函数值得出来的, 函数的极值是比较极值点附近的函数值得出来的。

\section{2. 可导函数的极值}

极值是一个局部性概念, 一个函数在其定义域内可以有许多个极大值和 极小值, 在某一点的极小值也可能大于另一点的极大值, 也就是说极大值与 极小值没有必然的大小关系。

若 $f(x)$ 在 $(a, b)$ 内有极值, 那么 $f(x)$ 在 $(a, b)$ 内绝不是单调函数, 即在 某区间上单调递增或减的函数没有极值。

\section{表 1. 极值和最值的知识点比较分析表}

\begin{tabular}{|c|c|c|c|c|}
\hline 极值 & 领域内的最值 & 不可导点或驻点 & 局部空间的最值 & 短期的最值 \\
\hline 最值 & $\begin{array}{c}\text { 函数给定区间的 } \\
\text { 最值 }\end{array}$ & $\begin{array}{c}\text { 不可导点、驻点和区 } \\
\text { 间端点 }\end{array}$ & 全体空间的最值 & 长远的最值 \\
\hline
\end{tabular}




\section{4. 极值和最值的数学思想}

极值和最值的关系是部分和整体的关系。整体和部分, 又称全局和局部, 是客观事物普遍联系的一种形式。它们是对立统一的关系。局部离不开整体, 离开了整体, 就失去了作为整体中的局部的性质和意义。整体功能大于各个 部分功能之和。条件是各部分必须以有序、合理、优化的结构形成整体。所 以全局大于局部, 统帅局部, 局部服从全局。整体与部分是相互依赖, 不可 分割, 各以对方存在为前提。整体由部分构成, 它只在对于组成它的部分而 言, 才是一个确定的整体。部分是整体中的部分, 没有整体也无所谓部分。

函数的最大值和最小值是一个整体性概念, 最大值必须是整个区间上所 有函数值中的最大值, 最小值必须是函数在整个定义区间上所有函数值中的 最小值。函数的最大值、最小值是比较整个定义区间的函数值得出来的, 函 数的极值是比较极值点附近一个较小的领域区间的函数值得出来的. 函数的 极大值或极小值可以有多有少, 但最大值或最小值只有一个; 极值只能在区 间内取得, 最值则可以在端点处取得; 有极值的未必有最值, 有最值的未必 有极值; 极值可能成为最值, 最值只要不在端点必定是极值。闭区间上的连 续函数一定有最大值和最小值, 开区间上的连续函数不一定有最大值和最小 值. 以导数为工具求函数的最值, 先找到极值点, 再求极值和区间端点函数 值, 其中最大的一个是最大值, 最小的一个是最小值。

极值和最值的关系是辩证统一的。函数的极值有可能成为函数的最值, 而函数的最值在较大发展空间(即定义域)也有可能成为极值而不是最值。因 此, 在学习函数的极值知识点的基础上, 要深入理解极值和最值之间的辩证 统一关系, 运用马克思主义发展和运动观点处理学习和工作中面临的极值和 最值问题，丰富和提高自己的思想内涵。

\section{5. 思政内涵解读}

\section{1. 极值和最值中的人生哲理一一寓道于教}

曲折起伏是人生的常态。一个人从呱呱坠地到灰飞烟灭, 不过百十年的 生命之旅, 若把人生看成路途, 那么人生路途中每迈出一步在生命星河中会 留下痕迹, 连缀起来的痕迹便是生命之旅在时空屏幕上划出的轨迹。这轨迹 是曲线，永远不可能是直线。人生毕竟是分阶段的，就如同分段函数，有美 满(短暂极大值)也有辛苦(暂时极小值), 有欢欣(瞬态极大值)也有苦痛(极小 值)。这说明人生中存在着函数的极值规律。人生就是这样, 正是因为其曲折, 屈伸才更多了几多回味, 才更显得其丰富, 直线纵然简练, 但却无法与曲线 之美相比。罗曼・罗兰说过一句话: “人生不是旅行, 不出售来回票, 一旦 动身就很难返回。”人生道路不可重复, 即使可以使自己重新开始, 但社会 在发展, 环境在变化, 所谓的 “重新开始” 也不是当初的 “重新开始”, 因为 根本不可能再面对同样的选择条件。数学老师在讲到 “函数的极值、最值” 这 部分内容时, 可以与思政教育中的人生教育内容相结合, 从而帮助学生认识到 人生由于函数变化, 既有高峰也有低谷。在漫长的人生道路上每个人都不可能 一帆风顺, 这就需要学生能够树立起健全的人格, 正确认识成功与失败。 


\section{2. 从极值和最值的视角谈国家利益、集体利益和个人利益一 育德于教}

如表 2 所示, 如果把极大值比作个人利益, 即是区域较小的利益, 那么 最大值就是国家利益和民族利益, 就是区域最大的利益(如图 2 所示)。2020 年的抗击新冠状肺炎, 再次证明, 国家利益和个人利益是统一的、一致的。 有些人为了个人舌尖的享受, 罔顾国家法律, 暂时满足了个人的口舌之欲, 其结果是既损害了国家利益, 个人利益也不能够得到安全保证。与之形成鲜 明对比的是那些白衣天使, 舍生忘死, 不顾个人安危, 冲在防疫抗疫的最前 线, 牺牲了个人的利益, 换来了整个中华民族的利益。

早在 1956 年, 毛泽东同志就曾在《论十大关系》中提出, 把集体主义作 为社会主义社会的基本原则 [3]。邓小平认为要辩证地看待这两者的关系, “在 社会主义制度之下, 个人利益要服从集体利益, 局部利益要服从整体利益, 暂时利益要服从长远利益, 或者叫做小局服从大局, 小道理服从大道理。我 们提倡和实行这些原则, 决不是说可以不注意个人利益, 不注意局部利益, 不注意暂时利益, 而是因为在社会主义制度之下, 归根结底, 个人利益和集 体利益是统一的, 局部利益和整体利益是统一的, 暂时利益和长远利益是统 一的。我们必须按照统筹兼顾的原则来调节各种利益的相互关系。如果相反, 违反集体利益而追求个人利益, 违反整体利益而追求局部利益, 违反长远利 益而追求暂时利益, 那末, 结果势必两头都受损失。” 并强调: “要防止盲 目性, 特别要防止只顾本位利益、个人利益而损害国家利益、人民利益的破 坏性的自发倾向。”[4]

2019 年 10 月适应新时代的新要求, 中共中央、国务院印发了《新时代公 民道德建设实施纲要》。在新时代公民道德建设中的总体要求里面提到要 “以 集体主义为原则” , 具有深远的历史意义和现实意义。集体主义作为道德原 则, 具有正确处理个人利益、集体利益与国家利益关系的重要作用。集体主 义原则提倡在国家利益、集体利益与个人利益发生矛盾时, 集体利益优于个 人利益, 国家利益又高于集体利益, 个人要以集体利益和国家利益为重, 而 且在必要的情况下, 个人应当为集体利益和国家利益放弃或牺牲个人利益。 与此同时, 集体主义原则又要求集体和国家必须尽力保障个人的正当利益能 够得到满足, 促进个人自由全面发展和人生价值的实现。从根本上说, 个人 利益与集体利益、国家利益是相辅相成、辩证发展的, 一方面, 没有集体和 国家的富强, 就不能切实保障个人的发展; 另一方面, 集体和国家又因为个 体的能动性得到充分发挥, 才能成为充满活力、具有创新能力、富有蓬勃朝 气的集体和国家。

\section{3. 从极值和最值的视角谈新发展理念一一寓教于乐}

2015 年 10 月 29 日习近平总书记在《中共中央关于制定国民经济和社会

表 2. 函数最值、及其和国家利益等之间的适应关系

\begin{tabular}{ccccc}
\hline 函数极值 & 个人利益 & 家庭利益 & 团体利益 & 机构利益 \\
函数最值 & 国家利益 & 集体利益 & 民族利益 & 人类共同体利益 \\
\hline
\end{tabular}




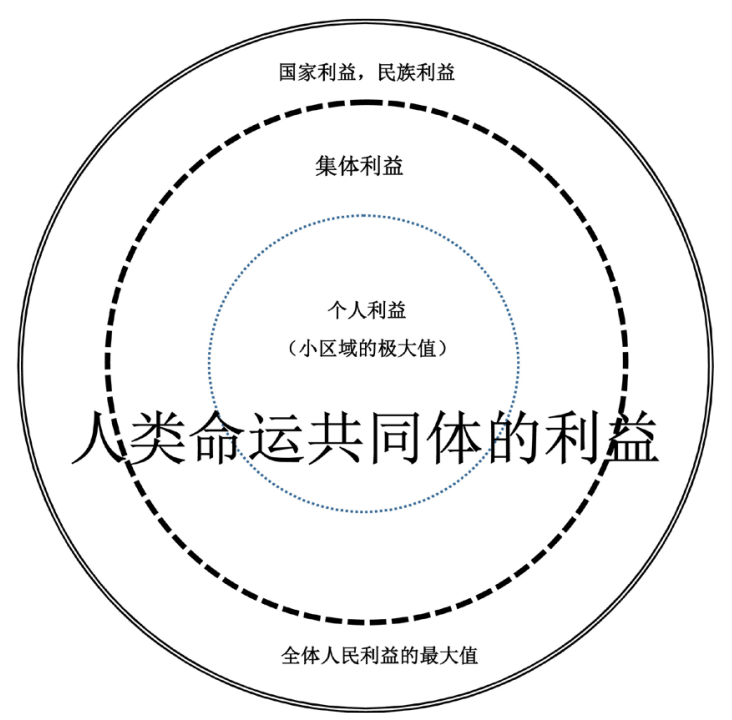

图 2. 个人利益、集体利益、国家利益、人类命运共 同体之间的关系

发展第十三个五年规划的建议》中强调, 首先要把应该树立什么样的发展理 念搞清楚…… 《建议》提出要坚持创新、协调、绿色、开放、共享的发展 理念 [5]。我们称之为新的发展理念, 其中绿色发展, 为千秋万代负责, 不欠 子孙债, 留下绿水青山。绿色发展注重解决的是人与自然的和谐问题, 其实 质就是人的发展极大值和自然环境的极大值要和谐。不可否认，在一段时间 里, 一些地方确实只片面地追求 GDP 的数量和速度。这种对 GDP 的盲目推 崇和追求，很大程度上反映在以浪费资源、破坏环境、损害群众利益等为突 出现象的粗放式增长方式上[6]。用数学的观点来看就是仅仅考虑了地方的经 济利益的极大值, 忽落了生态利益的极大值, 最终损害的不但是本地方可持 续发展的极大值, 而且对于国家的可持续发展造成了不良的影响。这样的片 面追求 GDP 极大值的发展方式无异于饮䴓止渴, 损害了子孙后代的发展权利 和利益, 如此的恶性循环最终会伤及人类自身。我们要牢记生态环境没有替 代品, 用新的发展理念指导国家的发展和进步, 进而实现中华民族的伟大复 兴的中国梦。

2016 年 1 月 18 日习近平总书记在省部级主要领导干部学习贯彻党的十八 届五中全会精神专题研讨班上讲话时对新发展理念作了更加深入的阐释。习 总书记指出, 下好 “十三五” 时期发展的全国一盘棋, 协调发展是制胜的要 诀。……, 处理好局部和全局、当前和长远、重点和非重点的关系, 在权衡 利弊中趋利避害、作出最为有利的战略抉择 [7]。正如我们就极值的数学思想 所做的论述，极值和最值的辩证关系，本身就是局部和全局、当前和长远的 关系, 如果我们能够在课堂教学中引领学生领悟新发展理念的美, 一定能够 在学生中产生共鸣的智慧。

\section{4. 从极值和最值的视角谈伟大的抗疫精神一( 元素化合、专题嵌入 和画龙点睛)}

结合当下的抗疫实践，伟大的抗疫精神更是深刻的折射出极值的思想隐 
喻。生命至上、举国同心、舍生忘死、尊重科学、命运与共的伟大抗疫精神。 生命至上集中体现了中国人民深厚的仁爱传统和中国共产党人以人民为中心 的价值追求, 这是中国共产党执政为民理念的最好诠释, 是中华文明人命关 天的道德观念的最好体现, 也是中国人民敬仰生命的人文精神的最好印证; 举国同心集中体现了中国人民万众一心、同甘共苦的团结伟力。长城内外、 大江南北, 全国人民心往一处想、劲往一处使, 把个人冷暖、集体荣辱、国 家安危融为一体。

通过深入剖析极值的思想价值和思维内涵, 透析伟大抗疫精神中思想, 我们会深刻感悟抗疫精神同中华民族长期形成的特质禀赋和文化基因一脉相 承, 是爱国主义、集体主义、社会主义精神的传承和发展, 是中国精神的生 动诠释, 丰富了民族精神和时代精神的内涵。我们要在全社会大力弘扬伟大 抗疫精神, 使之转化为全面建设社会主义现代化国家、实现中华民族伟大复 兴的强大力量。

\section{6. 结论}

数学揭示的是客观事物的普遍规律, 数学中利用导数求极值问题包含了 丰富的哲学思想和人生智慧。站在时代的高度, 融汇贯通 “三寓三式” 于极 值知识点的深入挖掘, 帮助学生体会如何在新时代做到个人利益、集体利益 和国家利益的统一, 如何正确看待人生道路上的利益得失, 引领学生领悟极 值中包含的科学辩证法和方法论, 帮助学生建立正确的义利观。在科技快速 发展的今天, 如何正确看待和处理专业发展利益和个人现实利益之间的关系 对于莘莘学子和思想发展关键期的青年人具有非常重要的意义, 本项目将会 结合学生专业进行深入探究。

\section{致谢}

本文受 2018 年度教育部高校示范马克思主义学院和优秀教学科研团队 建设项目 (18JDSZK012)、上海出版印刷高等专科学校 “课中课” 国家级教学 成果奖应用推广工程子项目 (ZK-2020-032), 2020 年上海出版印刷高等专科 学校思政重点课题和 2020 年上海出版印刷高等专科学校高教研究课题 (GJYJ-2020-10)资助。

\section{Conflicts of Interest}

The authors declare no conflicts of interest regarding the publication of this paper.

\section{References}

[1] 滕跃民, 张玉华, 肖纲领. 高职专业“课程思政”的“道法术器”改革 [J]. 辽宁高职 学报, 2018(8): 53-55.

[2] 滕跃民, 张玉华, 马前锋, 汪军, 孟仁振. 同向同行: 知识传授与价值引领同频共 振一一上海出版印刷高等专科学校“课中课”课程思政改革探析 $[\mathrm{N}]$. 中国教育报, 2019-06-19(11).

[3] 毛泽东. 论十大关系 $[M] / /$ 中央文献研究室编辑. 《毛泽东文集》第七卷. 北京: 
1999.

[4] 邓小平. 《邓小平文选》第 2 卷 $[\mathrm{M}]$. 北京: 人民出版社, 1994.

[5] 习近平. 以新的发展理念引领发展 $[\mathrm{M}] / /$ 《习近平谈治国理政》第二卷. 北京: 外 文出版社, 2017: 198.

[6] 毛晓刚. 应以科学理性的精神看待 GDP [J]. 领导科学, 2010(24): 18-19.

[7] 习近平. 深入理解新发展理念 $[\mathrm{M}] / /$ 《习近平谈治国理政》第二卷. 北京: 外文出 版社, 2017: 206.

\section{Appendix (Abstract and Keywords in Chinese)}

\section{高等数学极值的课程思政三寓三式探究}

摘要: 本文立足高等数学的极值教学内容, 通过三寓三式将知识教学与 德育熏染融于一体，通过对极值知识点的思想价值阐释，将知识传授与价值 引领有机融合, 引导学生正确做人做事做学问, 助力学生的全面发展。

关键词: 高等数学, 极值, 课程思政 